\title{
Reliability-Based Marginal Cost Pricing Problem Case with Both Demand Uncertainty and Travelers' Perception Errors
}

\author{
Shaopeng Zhong, ${ }^{1}$ Lihui Zhang, ${ }^{1}$ and Max Bushell ${ }^{2}$ \\ ${ }^{1}$ School of Transportation and Logistics, Dalian University of Technology, Dalian 116024, China \\ ${ }^{2}$ Pedestrian and Bicycle Information Center, University of North Carolina at Chapel Hill, Chapel Hill, NC 27514, USA \\ Correspondence should be addressed to Shaopeng Zhong; szsp001@163.com
}

Received 11 April 2013; Accepted 25 July 2013

Academic Editor: Jyh-Horng Chou

Copyright (c) 2013 Shaopeng Zhong et al. This is an open access article distributed under the Creative Commons Attribution License, which permits unrestricted use, distribution, and reproduction in any medium, provided the original work is properly cited.

\begin{abstract}
Focusing on the first-best marginal cost pricing (MCP) in a stochastic network with both travel demand uncertainty and stochastic perception errors within the travelers' route choice decision processes, this paper develops a perceived risk-based stochastic network marginal cost pricing (PRSN-MCP) model. Numerical examples based on an integrated method combining the moment analysis approach, the fitting distribution method, and the reliability measures are also provided to demonstrate the importance and properties of the proposed model. The main finding is that ignoring the effect of travel time reliability and travelers' perception errors may significantly reduce the performance of the first-best MCP tolls, especially under high travelers' confidence and network congestion levels. The analysis result could also enhance our understanding of (1) the effect of stochastic perception error (SPE) on the perceived travel time distribution and the components of road toll; (2) the effect of road toll on the actual travel time distribution and its reliability measures; (3) the effect of road toll on the total network travel time distribution and its statistics; and (4) the effect of travel demand level and the value of reliability (VoR) level on the components of road toll.
\end{abstract}

\section{Introduction}

It is well known that in the case of a deterministic networkdeterministic user equilibrium (DN-DUE) assignment, a user equilibrium (UE) flow pattern can be driven towards a system optimal (SO) flow pattern by replacing link cost-flow functions with marginal cost-flow functions. The underlined assumptions of the deterministic user equilibrium model are that travelers are assumed to know exactly the time on each available route and can always choose the least-cost routes for their trips. However, due to their physiological limitations, travelers may not have perfect knowledge about the road traffic condition, particularly in a congested network. Scholars gradually become aware that perceptions are important for accurate quantification of route travel time and the behavioral responses of travelers' route choice decisions. Therefore, more and more scholars have argued that the assumptions of the DN-DUE model are unrealistic and that the stochastic user equilibrium (SUE) model is more aligned with reality.
To account for the travelers' perception errors, researchers usually assume the commonly adopted Gumbel variate as the random error term and use the conventional logitbased stochastic user equilibrium (SUE) model. In the case of a deterministic network-stochastic user equilibrium (DNSUE) assignment, Yang [1] proposed a perceived deterministic network-marginal cost pricing (PDN-MCP) model, and he further pointed out that though the marginal cost pricing for a logit-based DN-SUE can reduce the total network travel cost (TNTC), it generally cannot achieve the minimum TNTC. It was later proven that marginal cost pricing in the DN-SUE case yields the deterministic network-stochastic system optimal (DN-SSO) solution instead of the actual total network travel cost being minimized. In fact, the "total perceived network travel cost" is minimized [2]. Actually, the perception error defined in the traditional logit-based SUE models can be regarded as "deterministic," because this kind of perception error is independent of the stochastic travel time (i.e., actual travel time distribution). In view of 
this, Mirchandani and Soroush [3] investigated the stochastic perception error traffic assignment problem. The stochastic perception error (SPE) is conditionally dependent on the actual travel time distribution, which is different from the deterministic perception error commonly adopted in the traditional logit-based SUE models by simply adding a random error term such as the Gumbel distribution to the route costs. Though the SPEs are important for accurate quantification of route travel time and the behavioral responses of travelers' route choice decisions, to the best of our knowledge, almost nothing has been published on the topic of integrated consideration of both marginal cost pricing and stochastic perception error.

Moreover, the first-best marginal cost pricing (MCP) is commonly modeled via a deterministic approach in which the route travel times are deterministic. Such an approach does not explicitly consider the value of reduced risk of high travel delay variance [4]. From a deterministic perspective, travelers are usually assumed to take only travel time and tolls into account when making travel route choice decisions. Trip reliability, as influenced by traffic accidents or flow changes, is not taken into account. However, due to stochastic variations in both supply and demand, the travel time almost always involves a measure of uncertainty. Recently, several empirical studies on the value of time and reliability revealed that travel time reliability plays an important role in the travelers' route choice decision-making process [5-7]. With these studies as a basis, the study of travel time variability (reliability) has gradually emerged as an important topic. In this context, travel time reliability pertains to the probability that a trip can be successfully completed within a specified time interval, reflecting the uncertainty in trip journey times $[8,9]$. To model the characteristics of travel time reliability, the concept of TTB is commonly used. TTB is defined as the average travel time plus extra time (for a measure of the buffer time) such that the probability of completing the trip within the TTB is no less than a predefined reliability threshold $\alpha$ [10]. Earlier research, by Uchida and Iida [11], applied the concept of effective travel time to capture the travel time reliability. More recently, Chen and Zhou [10] further proposed a stochastic mean-excess traffic equilibrium model to represent both the reliability and unreliability aspects of travel time variability and travelers' route choice perception errors.

Although the aforementioned studies discovered that travelers do indeed consider travel time variability as a risk in their route choice decisions, relatively little research has been done on the combination of marginal cost pricing and travel time reliability, especially regarding travelers' risk attitudes and/or the valuation of reliable travel under road congestion charge condition [12]. Boyles et al. [12] proposed a firstbest congestion pricing model considering network capacity uncertainty and user valuation of travel time reliability, while Sumalee and $\mathrm{Xu}[13]$ investigated the relationship between the stochastic network-user equilibrium (SN-UE) and stochastic network-system optimal ( $\mathrm{SN}-\mathrm{SO}$ ) models and established the stochastic network-marginal cost pricing (SN-MCP) scheme for a stochastic traffic network in which demand uncertainty is explicitly considered. The SN-MCP model represents a risk-neutral case in which travelers are only concerned about the average travel time when making route choice decisions. Under travel time uncertainty, travelers actually consider both travel time variability and mean travel time. Therefore, Sumalee and $\mathrm{Xu}$ [13] further derived the risk-based SNMCP (RSN-MCP) model in which travelers are assumed to consider both the mean travel time and reliability in their route choice decision. The RSN-MCP model aims to minimize the weighted sum of the mean and the variance of the total travel time, and it can describe travelers' risk-based (averse) behavior. Recently, Gardner et al. [14] considered the uncertainty in long-term travel demand and in day-today network capacity and discussed the benefit of responsive pricing and travel information.

All in all, the combination of the concept of the SPE and the RSN-MCP has not been explored in depth such as how to integrate travel time reliability and travelers' stochastic perception errors into the traditional congestion pricing model and simulate travelers' route choice behaviors under a tolled stochastic environment; how the SPE, travel demand level, and the value of reliability level influence the components of road toll; furthermore, how the road toll impacts the network performance and travelers' route choice behaviors under a congested risky circumstance. Exploring all the above questions is a key foundation for developing optimum congestion pricing strategies aimed at improving the efficiency and reliability of an urban stochastic transportation network. As a consequence of the above considerations, in this paper, we develop a generalized perceived risk-based stochastic network-marginal cost pricing (PRSNMCP) model to explicitly consider both the travel time variability and the travelers' stochastic perception errors of the random travel time. Note that, in this paper, we consider a stochastic traffic network problem due to stochastic travel demand. To facilitate the understanding of the model, following Chen et al's classification [15], a detailed classification of marginal cost pricing models in the literature is provided in Table 1 based on (1) perception errors due to the traveler's imperfect information with regard to travel time distribution and (2) network uncertainty caused by the stochasticity of network travel times.

The following are the main contributions of this paper.

First, in order to explicitly consider both the travel time variability and the travelers' stochastic perception errors of the random travel time, the paper develops a generalized perceived risk-based stochastic network-marginal cost pricing (PRSN-MCP) model. The PRSN-MCP model presented here can be considered a generalization of the MCP model in a stochastic network. The PRSN-MCP model can be reduced to a certain "incomplete" marginal cost pricing model when certain aspect of the PRSN-MCP model is ignored. For example, if the travelers' stochastic perception errors are neglected, then the PRSN-MCP model is reduced to the RSN-MCP model proposed by Sumalee and Xu [13]. If both the travelers' perception errors and travel time reliability are ignored, then the PRSN-MCP model is reduced to the SNMCP model.

Second, based on the proposed PRSN-MCP model, this study investigates thr following: (1) the effect of SPE on the perceived travel time distribution and the components of 
TABLE 1: Classification of marginal cost pricing models in the literature.

\begin{tabular}{llccc}
\hline & & & Perception error? & Yes \\
& & No & Deterministic & Stochastic \\
\hline \multirow{2}{*}{ Network Uncertainty? } & No & DN-MCP & PDN-MCP & - \\
& Yes & SN-MCP & - & PRSN-MCP (this paper) \\
\hline
\end{tabular}

DN: deterministic network; SN: stochastic network; RSN: risk-based stochastic network; RDN: perceived deterministic network; PRSN: perceived risk-based stochastic network; MCP: marginal cost pricing.

TABLE 2: Acronyms used in the paper.

\begin{tabular}{ll}
\hline Abbreviation & Complete form \\
\hline BTTRT & Buffer travel time-related toll \\
DN-DUE & $\begin{array}{l}\text { Deterministic network-deterministic user } \\
\text { equilibrium }\end{array}$ \\
DN-MCP & Deterministic network-marginal cost pricing \\
DN-SSO & Deterministic network-stochastic system \\
& optimal \\
DN-SUE & Deterministic network-stochastic user \\
FDM & equilibrium \\
MCP & Fitting distribution method \\
MTTRT & Marginal cost pricing \\
PDN-MCP & Mean travel time related toll \\
& Perceived deterministic network-marginal cost \\
pricing \\
PRS-MCP & Perceived risk-based stochastic \\
PRSN-SO & Petwork-marginal cost pricing \\
& network-system optimal \\
RSN-MCP & Risk-based stochastic network-marginal cost \\
Pricing & Perceived travel time \\
SN-MCP & Stochastic network-marginal cost pricing \\
SN-SO & Stochastic network-system optimal \\
SN-UE & Stochastic network-user equilibrium \\
SPE & Stochastic perception error \\
TTB & Travel time budget \\
TTD & Travel time distribution \\
VMR & Total travel time \\
\hline & Variance-to-mean ratio \\
&
\end{tabular}

road toll; (2) the effect of road toll on the actual travel time distribution and its reliability measures; (3) the effect of road toll on the total network travel time distribution and its statistics; and (4) the effect of travel demand level and the value of reliability (VoR) level on the components of road toll.

Third, the current paper develops these results with an approach that differs from that in the literature. This paper uses an integrated method combining the moment analysis approach, the fitting distribution method, and the reliability measures. The fitting distribution method is used to fit the moments obtained from the moment analysis approach, and the resulted distribution curves can provide a more intuitive "picture" of distribution. On the other hand, reliability measures can provide further a more quantitative characterization of the distribution. Hence, the analysis objectives and analysis tool are both different from that in the literature.

The remainder of the paper is organized as follows. The next section introduces the assumptions used in the analysis and presents the variational inequality (VI) formulation for the perceived risk-based stochastic network-system optimal (PRSN-SO) model. Then, Section 3 derives the analytical function of PRSN-MCP model. The fitting distribution method and reliability measures are used simultaneously to explore the effects of SPE and road toll in Section 4. The final section contains some concluding remarks and recommends further research. Acronyms used in this paper are summarized in Table 2.

\section{Framework of Stochastic Network Model}

2.1. Notations and Assumptions. Consider a strongly connected network $G=(N, A)$, where $N$ is the set of nodes and $A$ is the set of links in the network. Let $W$ represent the set of OD pairs in the network, and let the set of routes between OD pair $w \in W$ be denoted by $R_{w}$. The notations used in the paper are summarized in Table 3. For consistency, random variables are expressed in capital letters and lower-case letters are used for mean values of random variables or deterministic variables. Variables with a superscript “ $~ "$ " represent perceived variables.

Before proceeding with the analysis, some assumptions are made to allow for the closed-form formulation/calculation of the PRSN-MCP model.

(A1) The travel demands $Q^{w}$ between each OD pair are assumed to be an independent random variable with a mean of $q^{w}$ and variance of $\varepsilon_{q}^{w}$, while $\mathrm{VMR}_{w}$ is the variance-to-mean ratio (VMR) of the random travel demand in which $\mathrm{VMR}_{w}=\varepsilon_{q}^{w} / q^{w}$. Stochastic demand is further assumed to follow a lognormal distribution, which is a nonnegative, asymmetrical distribution. This has been adopted in the literature as a more realistic approximation of the stochastic travel demand, as opposed to the more commonly used normal distribution $[13,16]$.

(A2) The route flow $F_{r}^{w}$ and link flow $V_{a}$ are also assumed to be independent random variables that follow the 
TABLE 3: A summary of notations.

\begin{tabular}{|c|c|}
\hline Notations & Explanations \\
\hline$Q^{w}$ & Travel demand between OD pair $w \in W$ \\
\hline$q^{w}$ & Mean travel demand between OD pair $w \in W$ \\
\hline$\varepsilon_{q}^{w}$ & $\begin{array}{l}\text { Variance of travel demand between OD pair } \\
w \in W\end{array}$ \\
\hline $\mathrm{VMR}_{w}$ & $\begin{array}{l}\text { Variance-to-mean ratio (VMR) of the random } \\
\text { travel demand }\end{array}$ \\
\hline$F_{r}^{w}$ & Route flow on path $r \in R_{w}$ \\
\hline$f_{r}^{w}$ & Mean traffic flow on path $r \in R_{w}$ \\
\hline$\varepsilon_{f}^{w, r}$ & Variance of traffic flow on path $r \in R_{w}$ \\
\hline f & $\begin{array}{l}\text { Column vector of mean route flow, where } \\
\mathbf{f}=\left\{f_{r}^{w}\right\}\end{array}$ \\
\hline$V_{a}$ & Traffic flow on link $a \in A$ \\
\hline$v_{a}$ & Mean traffic flow on link $a \in A$ \\
\hline$\varepsilon_{v}^{a}$ & Variance of traffic flow on link $a \in A$ \\
\hline $\mathbf{v}$ & Column vector of mean link flow, where $\mathbf{v}=\left\{v_{a}\right\}$ \\
\hline$\delta_{a, r}^{w}$ & $\begin{array}{l}\text { Link-path incidence parameter; } 1 \text { if link } a \text { on path } \\
r, \text { zero otherwise }\end{array}$ \\
\hline $\mathrm{TT}$ & $\begin{array}{l}\text { Total travel time of the system, where } \\
\mathrm{TT}=\sum_{a \in A} V_{a} T_{a}\end{array}$ \\
\hline VoR & $\begin{array}{l}\text { Relative weight assigned to the travel time budget, } \\
\text { that is, value of reliability }\end{array}$ \\
\hline$T_{a}$ & Travel time on link $a$ \\
\hline$t_{a}$ & Mean travel time on link $a$ \\
\hline$\widetilde{T}_{r}^{w}$ & Perceived travel time on path $r \in R_{w}$ \\
\hline$\vec{t}_{r}^{w}$ & Mean perceived travel time on path $r \in R_{w}$ \\
\hline$\widetilde{\varepsilon}_{t}^{w, r}$ & Variance of perceived travel time on path $r \in R_{w}$ \\
\hline$\widetilde{T}_{a}$ & Perceived travel time on link $a$ \\
\hline$\tilde{t}_{a}$ & Mean perceived travel time on link $a$ \\
\hline$\widetilde{\varepsilon}_{t}^{a}$ & Variance of perceived travel time on link $a \in A$ \\
\hline$\widetilde{T} \widetilde{T}$ & $\begin{array}{l}\text { Total perceived travel time of the system, where } \\
\widetilde{T} \widetilde{T}=\sum_{a \in A} V_{a} \widetilde{T}_{a}\end{array}$ \\
\hline$t_{a}^{0}$ & Free-flow travel time on link $a \in A$ \\
\hline$c_{a}$ & Capacity of link $a \in A$ \\
\hline$y_{a}$ & Parameter, where $y_{a}=\sqrt{1+\mathrm{VMR} / v_{a}}$ \\
\hline$\left.\varepsilon_{a}\right|_{T_{a}}$ & Travelers' perception errors on link $a \in A$ \\
\hline$N\left(\chi, \omega^{2}\right)$ & $\begin{array}{l}\text { Perception error distribution of traveler, in this } \\
\text { study } N\left(\chi, \omega^{2}\right) \text { follows a normal distribution } \\
\text { with predefined and deterministic mean } \chi \text { and } \\
\text { variance } \omega^{2}\end{array}$ \\
\hline
\end{tabular}

same statistical distribution as OD demand. The VMRs of route flows are equal to those of the corresponding OD demand.

(A3) The VMRs of travel demand are assumed to be the same for all OD pairs in order to derive the closedform formulation of the PRSN-MCP model.

(A4) The perception error distribution of an individual traveler for a segment of road with unit travel time equals $N\left(\chi, \omega^{2}\right)$, where $N\left(\chi, \omega^{2}\right)$ represents a normal distribution with predefined and deterministic mean $\chi$ and variance $\omega^{2}$.
(A5) Traveler's perception errors are independent for nonoverlapping route segments.

(A6) Traveler's perception errors are mutually independent over the population of travelers.

2.2. PRSN-SO Formulation. In this section, we describe the perceived risk-based stochastic network-system optimal (PRSN-SO) model for determining the equilibrium flow pattern under stochastic travel times and perception errors. Several empirical studies on the value of time and reliability reveal that travel time reliability plays an important role in the traveler's route choice decision process [5-7]. Therefore, we consider the risk-based (averse or prone) case in which travelers are assumed to consider both the mean travel time and travel time variability in their route decision-making process. In this study, we use the travel time budget (TTB) to represent travelers' risk-based travel behaviors. Besides, due to the imperfect knowledge about the network condition, travelers' perception errors have to be incorporated into their route choice decision process.

According to the assumptions (A1) and (A2), the OD travel demand $Q^{w}$, route flow $F_{r}^{w}$, and link flow $V_{a}$ are random variables, which consequently induce the random route/link travel times. As such, we have the following flow conservation relationships among them:

$$
\begin{gathered}
Q^{w}=\sum_{r \in R_{w}} F_{r}^{w}, \quad w \in W, \\
V_{a}=\sum_{w \in W} \sum_{r \in R_{w}} \delta_{a, r}^{w} F_{r}^{w}, \quad \forall a \in A, \\
F_{r}^{w} \geq 0, \quad w \in W, \quad r \in R_{w},
\end{gathered}
$$

where (1) is the travel demand conservation constraint, (2) is a definitional constraint that sums up all route flows that pass through a given link $a$, and (3) is a nonnegativity constraint on the route flows. Let $\Delta=\left[\delta_{a, r}^{w}\right]$ denote the route-link incidence matrix; $\delta_{a, r}^{w}=1$ if route $r$ traverses link $a$, and $\delta_{a, r}^{w}=0$ otherwise. Let $f_{r}^{w}, v_{a}$ denote the mean route flow and link flow, respectively. From (1) (3), these route and link flows satisfy the following conservation conditions:

$$
\begin{gathered}
q^{w}=\sum_{r \in R_{w}} f_{r}^{w}, \quad w \in W, \\
v_{a}=\sum_{w \in W} \sum_{r \in R_{w}} \delta_{a, r}^{w} f_{r}^{w}, \quad \forall a \in A, \\
f_{r}^{w} \geq 0, \quad w \in W, \quad r \in R_{w} .
\end{gathered}
$$

Let $\varepsilon_{f}^{w, r}, \varepsilon_{v}^{a}$ be the variance of route flow and link flow, respectively. Then, from the assumptions (A1) and (A2), we have

$$
\sum_{r \in R_{w}} \varepsilon_{f}^{w, r}=\sum_{r \in R_{w}} f_{r}^{w} \mathrm{VMR}_{w}=q^{w} \mathrm{VMR}_{w}=\varepsilon_{q}^{w}, \quad w \in W,
$$




$$
\begin{aligned}
\varepsilon_{v}^{a} & =\sum_{w \in W} \sum_{r \in R_{w}}\left(\delta_{a, r}^{w}\right)^{2} \varepsilon_{f}^{w, r}=\sum_{w \in W} \sum_{r \in R_{w}} \delta_{a, r}^{w} \varepsilon_{f}^{w, r} \\
& =\sum_{w \in W} \sum_{r \in R_{w}} \delta_{a, r}^{w} f_{r}^{w} \mathrm{VMR}_{w} .
\end{aligned}
$$

From (5) and (6), we know that the variances of both route flow and link flow can be determined by the means of route flows. Furthermore, the route and link flow distribution can be derived through known travel demand distributions.

In the following, we give the route and link travel disutility. Mathematically, the perceived TTB associated with route $r, \widetilde{b}_{r}^{w}$ is described as

$$
\widetilde{b}_{r}^{w}=E\left[\widetilde{T}_{r}^{w}\right]+\operatorname{VoR} \cdot \widetilde{\varepsilon}_{t}^{w, r}, \quad w \in W, r \in R_{w},
$$

where $\widetilde{\varepsilon}_{t}^{w, r}$ is the variance of the perceived route travel time, $\widetilde{T}_{r}^{w}$ is the perceived route travel time, and VoR is the relative weight assigned to the TTR, that is, value of reliability. Similarly, let $\widetilde{\varepsilon}_{t}^{a}$ be the variance of perceived link travel time, and let $\widetilde{T}_{a}$ be the perceived link travel time. The perceived TTB associated with link $a, \widetilde{b}_{a}$ can be described by

$$
\widetilde{b}_{a}=E\left[\widetilde{T}_{a}\right]+\operatorname{VoR} \cdot \widetilde{\varepsilon}_{t}^{a}, \quad a \in A
$$

Based on the assumption of independent travel time on each link, we can infer the following relationship between the variances of perceived route travel time and perceived link travel time as follows:

$$
\widetilde{\varepsilon}_{t}^{w, r}=\sum_{a \in A} \delta_{a, r}^{w} \widetilde{\varepsilon}_{t}^{a}, \quad w \in W, r \in R_{w}
$$

From (7) (9), the perceived TTB of the route and link satisfies the following conservation conditions:

$$
\begin{aligned}
\widetilde{b}_{r}^{w} & =E\left[\widetilde{T}_{r}^{w}\right]+\mathrm{VoR} \cdot \widetilde{\varepsilon}_{t}^{w, r} \\
& =\sum_{a \in A} \delta_{a, r}^{w} E\left[\widetilde{T}_{a}\right]+\mathrm{VoR} \cdot \sum_{a \in A} \delta_{a, r}^{w} \widetilde{\varepsilon}_{t}^{a} \\
& =\sum_{a \in A} \delta_{a, r}^{w} \widetilde{b}_{a}, \quad w \in W, \quad r \in R_{w} .
\end{aligned}
$$

We next investigate the formulation of the PRSN-SO model. The objective function of PRSN-SO should follow the value-at-risk concept, which is to minimize the weighted sum of the mean and the variance of the total perceived travel time. Therefore, the following mathematical program for PRSN-SO is defined:

$$
\min _{\mathbf{V}} W[\widetilde{T} \widetilde{T}]=E[\widetilde{T} \widetilde{T}]+\operatorname{VoR} \cdot \operatorname{Var}[\widetilde{T} \widetilde{T}]
$$

where $\widetilde{T} \widetilde{T}=\sum_{a \in A} V_{a} \widetilde{T}_{a}$. The constraints of (11) are the same as those of (4).

Therefore, the VI formulation for the link-based PRSNSO model can be expressed as

$$
\left(\mathbf{v}-\mathbf{v}^{*}\right)^{T} \nabla_{\mathbf{v}} W\left[\widetilde{T} \widetilde{T}^{*}\right] \geq 0
$$

s.t. (4), where $\nabla_{\mathbf{v}} W\left[\widetilde{T} \widetilde{T}^{*}\right]=\left\{\partial E\left[\sum_{a \in A} V_{a}^{*} \widetilde{T}_{a}^{*}\right] / \partial v_{a}^{*}+\operatorname{VoR}\right.$. $\left.\partial \operatorname{Var}\left[\sum_{a \in A} V_{a}^{*} \widetilde{T}_{a}^{*}\right] / \partial v_{a}^{*}\right\}$.

Proposition 1. The VI formulation for the PRSN-SO (see (11)) can be written as (12).

Proof. Note that the feasible set, defined by (4), is closed and convex; and the objective function in (11) is continuously differentiable on the feasible set. Then, according to Proposition 1.2 in Nagurney [17], the VI formulation for the PRSNSO problem (see (4) and (11)) can be written as (12). This completes the proof.

\section{Formulation of PRSN-MCP}

3.1. Derivation of PRSN-MCP Model. Let TT $=\sum_{\mathrm{a} \in \mathrm{A}} \mathrm{V}_{\mathrm{a}} \mathrm{T}_{\mathrm{a}}$; then the real gap between the marginal social and marginal private costs in a stochastic network can be represented by

$$
\mathrm{SN}-\mathrm{MCP}=\frac{\partial E\left[\sum_{a \in A} V_{a} T_{a}\right]}{\partial v_{a}}-E\left[T_{a}\right]=\frac{\partial E[\mathrm{TT}]}{\partial v_{a}}-E\left[T_{a}\right]
$$

Based on (11) (13), the PRSN-MCP toll can be given by

$$
\begin{aligned}
\text { PRSN-MCP }= & \left\{\frac{\partial E[\widetilde{T} \widetilde{T}]}{\partial v_{a}}-E\left[\widetilde{T}_{a}\right]\right\} \\
& +\operatorname{VoR} \cdot\left\{\frac{\partial \operatorname{Var}[\widetilde{T} \widetilde{T}]}{\partial v_{a}}-\operatorname{Var}\left[\widetilde{T}_{a}\right]\right\} .
\end{aligned}
$$

According to (14), it is clear that the value of PRSNMCP can be determined as long as $\partial E[\widetilde{T} \widetilde{T}] / \partial v_{a}, E\left[\widetilde{T}_{a}\right]$, $\partial \operatorname{Var}[\widetilde{T} \widetilde{T}] / \partial v_{a}$, and $\operatorname{Var}\left[\widetilde{T}_{a}\right]$ are known. From the conditional moment analysis, we can obtain $E\left[\widetilde{T}_{a}\right]$ and $\operatorname{Var}\left[\widetilde{T}_{a}\right]$ (see Appendix A for the derivations). Similarly, based on the moment analysis, we can derive the mean and variance of $\widetilde{T} \widetilde{T}$ (see Appendix B for the derivations). Substituting (A.5), (A.7), (B.2), and (B.4) into (14) and performing some derivation, we have

\section{PRSN-MCP}

$$
=\underbrace{(1+\chi)\left\{\partial E[\mathrm{TT}] / \partial v_{a}-E\left[T_{a}\right]\right\}}_{\text {mean travel time related toll }}+\underbrace{\operatorname{VoR} \cdot\left\{(1+\chi)^{2}\left\{\partial \operatorname{Var}[\mathrm{TT}] / \partial v_{a}-\operatorname{Var}\left[T_{a}\right]\right\}+\omega^{2}\left\{\partial E\left[V_{a}^{2} T_{a}\right] / \partial v_{a}-E\left[T_{a}\right]\right\}\right\} .}_{\text {buffer travel time related toll }}
$$

According to the assumption (A4), $\chi$ and $\omega^{2}$ represent the mean and the variance of an individual traveler's perception error distribution. Note that the PRSN-MCP model presented here can be considered a generalization of the MCP 
model in a stochastic network. The PRSN-MCP can be decomposed into two individual components, that is, the mean travel time-related toll (MTTRT) and the buffer travel time-related toll (BTTRT). If the VoR and $\chi$ are equal to zero, then the model in (15) is reduced to the SN-MCP model for a risk-neutral case. Besides, if the $\chi$ and $\omega$ are equal to zero, then the model in (15) is simplified as the RSN-MCP model.

\subsection{Calculation of PRSN-MCP Model under Lognormal} Demand. Due to the complexity of PRSN-MCP model, especially the introduction of the variance term in (15) prevents us from deriving a general closed form of the PRSNMCP toll. However, for calculation purposes, we can derive a specific closed-form PRSN-MCP toll for a certain statistical distribution of the travel demand. In this section, we present the specific form of the PRSN-MCP toll.

As discussed before, travel time variability may come from any combination of the variables $T_{a}^{0}$ (random link freeflow travel time), $V_{a}$ (random link flow induced by day-today travel demand variation), and/or $C_{a}$ (link capacity subject to stochastic degradation) [10]. For simplicity, we assume that the probabilistic link travel time $T_{a}$ only comes from the randomness of the travel demand. Therefore, in order to understand travel time variability, the characteristics of stochastic travel demand need to be clarified first.

Several types of probability distributions of OD travel demand have been adopted by researchers to simulate the travel demand fluctuation, such as normal distribution [18], lognormal distribution [16], and Poisson distribution [19]. As indicated in assumption (A1), we use the lognormal distribution in this study, which is more realistic than the commonly adopted normal distribution.

The probability density function of the lognormal distribution is given below:

$$
f(x \mid \mu, \sigma)=\frac{1}{x \sigma \sqrt{2 \pi}} \exp \left(\frac{-(\ln x-\mu)^{2}}{2 \sigma^{2}}\right), \quad \forall x>0,
$$

where $x$ is the random variable, $\mu$ and $\sigma$ are the distribution parameters, and the mean and variance of $x$ are $E[x]=$ $e^{\mu+\sigma^{2} / 2}$ and $\operatorname{Var}[x]=e^{2 \mu+\sigma^{2} / 2}\left(e^{\sigma^{2}}-1\right)$. Based on assumptions (A1) and (A2), with lognormal OD demand, the link flows also follow a lognormal distribution

$$
V_{a} \sim \ln \left(\mu_{v}^{a}, \sigma_{v}^{a}\right), \quad \forall a \in A,
$$

where $\mu_{v}^{a}=\ln \left(v_{a}\right)-(1 / 2) \ln \left(1+\varepsilon_{v}^{a} /\left(v_{a}\right)^{2}\right),\left(\sigma_{v}^{a}\right)^{2}=\ln (1+$ $\left.\varepsilon_{v}^{a} /\left(v_{a}\right)^{2}\right), v_{a}, \varepsilon_{v}^{a}$ are the mean and variance of link flow on link $a \in A$.

All of the moments of a lognormal random variable exist and are given as follows:

$$
E\left[X^{k}\right]=\exp \left(k \mu+\frac{k^{2} \sigma^{2}}{2}\right)
$$

where $E\left[X^{k}\right]$ is the $k$ th moment of $X$. With (6) and assumption (A3), we have

$$
\begin{aligned}
\varepsilon_{v}^{a} & =\sum_{w \in W} \sum_{r \in R_{w}}\left(\delta_{a, r}^{w}\right)^{2} \varepsilon_{f}^{w, r} \\
& =\mathrm{VMR} \cdot \sum_{w \in W} \sum_{r \in R_{w}} \delta_{a, r}^{w} f_{r}^{w} \\
& =\mathrm{VMR} \cdot v_{a}, \quad a \in A .
\end{aligned}
$$

Let $y_{a}=\sqrt{1+\mathrm{VMR} / v_{a}}$. Then, by using (16) (18) and performing some derivations according to Sumalee and $\mathrm{Xu}$ [13], we can obtain

$$
\begin{aligned}
E\left[V_{a}^{n}\right] & =\exp \left(n \mu_{v}^{a}+\frac{n^{2}\left(\sigma_{v}^{a}\right)^{2}}{2}\right)=v_{a}^{n}\left(\sqrt{\frac{1+\mathrm{VMR}}{v_{a}}}\right)^{n^{2}-n} \\
& =v_{a}^{n} y_{a}^{n^{2}-n}, \\
\operatorname{Var}\left[V_{a}^{n}\right] & =E\left[V_{a}^{2 n}\right]-\left(E\left[V_{a}^{n}\right]\right)^{2} \\
& =v_{a}^{2 n} y_{a}^{4 n^{2}-2 n}-v_{a}^{2 n} y_{a}^{2 n^{2}-2 n} .
\end{aligned}
$$

In this study, the link travel time function is assumed to be the Bureau of Public Roads (BPRs) function, $T_{a}=$ $t_{a}^{0}\left(1+\beta\left(V_{a} / c_{a}\right)^{n}\right)$, for all $a \in A$, where $T_{a}, t_{a}^{0}, c_{a}, V_{a}$ are the travel time, free-flow travel time, capacity, and traffic flow on link $a . \beta$ and $n$ are the deterministic parameters. According to (15), in order to calculate the value of PRSN-MCP, we need first to know the values of $E\left[T_{a}\right], \operatorname{Var}\left[T_{a}\right], \partial E[\mathrm{TT}] / \partial v_{a}, \partial \operatorname{Var}[\mathrm{TT}] / \partial v_{a}$, and $\partial E\left[V_{a}^{2} T_{a}\right] / \partial v_{a}$. Appendix $C$ provides the detailed derivation of these variables. Thus, the value of PRSN-MCP can be determined by substituting (B.4), (C.3), (C.4), (C.6), and (C.8) into (15).

\section{Numerical Examples}

The purposes of the numerical examples are to illustrate the following: (1) the effect of SPE on the perceived travel time distribution and the components of road toll; (2) the effect of road toll on the actual travel time distribution and its reliability measures; (3) the effect of road toll on the total network travel time distribution and its statistics; and (4) the effect of travel demand level and the VoR level on the components of road toll. The proposed models in this paper can be solved by the Method of Successive Averages (MSAs).

In order to enhance the understanding of the effects of the SPE (or road toll) and also the visualization on the travel time distribution, we use the fitting distribution method (FDM) to fit the moments obtained from the moment analysis approach. The FDM is able to reconstruct a full probability distribution for the travel time or the total network travel time. The interested readers may refer to Clark and Watling [19] and Hill et al. [20] for details of the FDM. On the other hand, we use four reliability measures to further explore the analytical and quantitative relationships between actual travel 

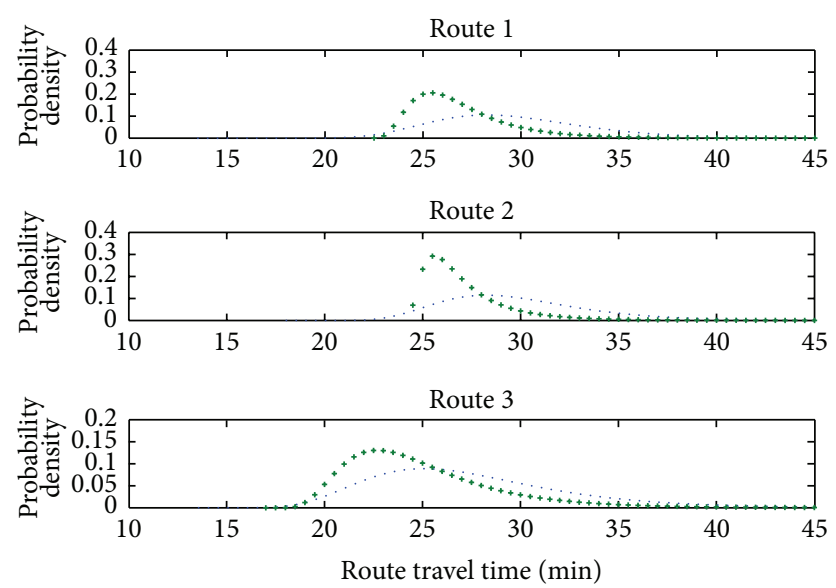

- Perceived travel time

+ Actual travel time

Figure 1: Probability of distribution of the actual and perceived travel times.

time reliability and road toll. The FDM and statistic measures are used simultaneously to complement each other.

For demonstration purpose, a simple network with three parallel routes (here links and routes are identical) is adopted to conduct a set of numerical experiments. There is one O-D pair $(1,2)$. The O-D demand is assumed to follow a lognormal distribution with a mean of 1000 flow units and a varianceto-mean ratio (VMR) of 6 . The link travel time function is assumed to be the Bureau of Public Roads (BPRs) function, which is $T_{a}=t_{a}^{0}\left(1+0.15\left(V_{a} / c_{a}\right)^{4}\right)$, for all $a \in A$. The freeflow travel times in minute of the three links are, respectively, 22,24 , and 17 . Their capacities are assumed to be, respectively, 350,220 , and 320 vehicles per minute (veh/min).

\subsection{Effect of SPE}

4.1.1. Effect on the Perceived Travel Time Distribution. From Figure 1, we can observe that with the consideration of stochastic perception error (SPE), the perceived TTDs are not only gradually moving to the right with a larger variability, but also becoming more dispersed and randomly compared to the actual TTDs. Therefore, it is clear that the SPE affects travelers' perception of the actual TTDs, and thus it can also significantly affect travelers' route choice decisions [21].

4.1.2. Effect on the Components of Road Toll. Next, we investigate the effect of SPE on the components of road toll. As discussed in Section 3, the road toll can be decomposed into two different components, that is, the average travel timerelated toll (MTTRT) and the buffer travel-time related toll (BTTRT). This can be demonstrated more clearly in Figure 3. For illustration purpose, we only show route 1 in Figure 2.

From Figure 2, we can see the following.

(i) The SPE mean has a significant effect on the MTTRT, while its variance has no effect on the MTTRT. For the BTTRT, the effect of mean is larger than that of

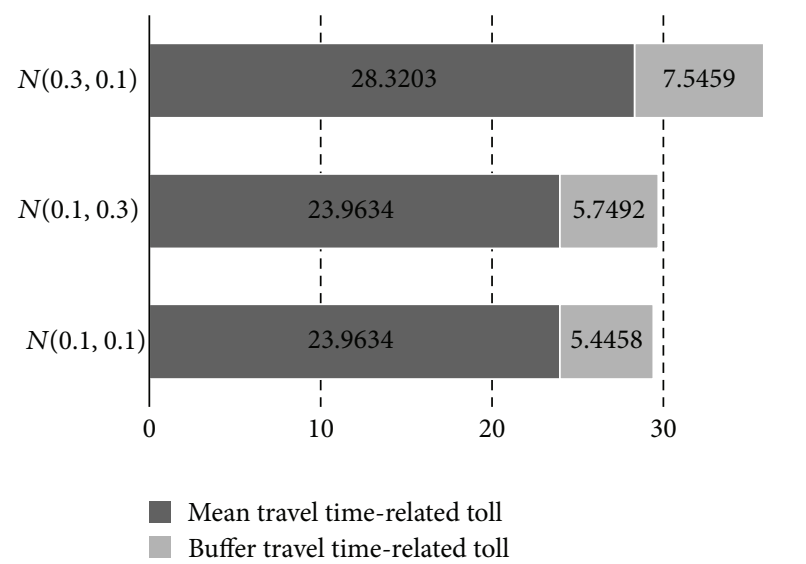

FIGURE 2: Effect of SPE on the components of road toll on route 1.

variance. These results seem to imply that the SPE mean plays a more important role on the different components of road toll.

(ii) With the increase of mean and variance, the road toll (the sum of MTTRT and BTTRT) is increasing. A reasonable explanation for this phenomenon is that when travelers' perception on travel times becomes more inaccurate, in order to arrive on time at a given confidence level, travelers need to budget a large buffer time, which is leading to a higher rate of congestion charges.

4.2. Effect of Road Toll. In this section, we examine the effect of road toll at two levels: (1) individual actual travel time distribution (TTD) and its reliability measures; (2) total network travel time distribution and its statistics. It should be noted that tolls are charged on all the links.

4.2.1. Effect on the Actual Travel Time Distribution and Its Reliability Measures. First, Figure 3 shows the actual travel time distribution (cumulative probability and probability density) patterns before and after the implementation of link tolls. From Figure 3, the following observations can be drawn:

(i) behavioral responses of travelers are explicitly captured in the proposed model. As shown in Figure 3(a), actual travel time distribution on link 3 is reduced (shifted to the left) when the road toll is implemented. In contrast, actual travel time distribution on link 1 and link 2 is increased (shifted to the right);

(ii) at first glance, with the introduction of the road toll, the probability distribution of the actual travel times of all three routes becomes more concentrated and skewed compared to the toll-free case. That implies that descriptive statistics such as mean and variance (standard deviation) are not very useful in describing the asymmetric actual travel time distribution or as indicators of reliability $[22,23]$. Therefore, mean and variance (standard deviation) are not appropriate 
TABLE 4: Actual travel time reliability measures.

\begin{tabular}{lccccc}
\hline Measure & \multicolumn{2}{c}{ Toll } & \multicolumn{3}{c}{ Toll-free } \\
\hline Route number & 1 & 2 & 3 & 1 & 2 \\
$\lambda^{\text {skew }}:(T 90-T 50) /(T 50-T 10)$ & 1.8929 & 2.2876 & $\mathbf{1 . 8 8 8 4}$ & 1.9082 & 2.3878 \\
$\lambda^{\text {var }}:(T 90-T 10) / T 50$ & 0.2421 & 0.2650 & 0.3159 & 0.2282 & 0.1877 \\
Buffer index: (T95-Tmean)/Tmean & 0.1970 & 0.2301 & 0.2558 & 0.1864 & 0.1674 \\
Planning time index: T95/T15 & 1.3190 & 1.3662 & 1.4272 & 1.2999 & 0.3860 \\
\hline
\end{tabular}

TXX denotes the XX percentile value; Tmean is the average travel time.

measures for analyzing the effect of road toll on the reliability of actual travel time.

Second, we adopt the following four reliability measures recommended by the US Department of Transportation (DOT) guide and van Lint and van Zuylen [23] to further explore the analytical and quantitative relationships between actual travel time reliability and road toll. These measures is as are $\lambda^{\text {skew }}, \lambda^{\mathrm{var}}$, buffer index, and planning time index. A brief introduction to these reliability measures is as follows: $\lambda^{\text {skew }}$ is defined as the ratio of the difference between the 90th percentile travel time and the median and the difference between the median and the 10th percentile [23]. In general, for very large $\lambda^{\text {skew }}$, the distribution is strongly left skewed, which means that the travel time distribution is unreliable. $\lambda^{\mathrm{var}}$ is used to describe the width of travel time distribution; large values indicate that the width of the distribution is large relative to its median value, and, hence, actual travel time may be interpreted as unreliable. Buffer index is the extra time required to arrive on time in addition to the average travel time. Here, the buffer index is defined by the difference between the 95th percentile travel time and the average travel time, normalized by the average travel time. The larger the value of buffer index, the lower actual travel time reliability. The planning time index is the ratio of the 95th percentile travel time over free-flow travel time (the 15th percentile travel time is considered as free-flow travel time in this study). A large value of the planning time index means low actual travel time reliability.

From Table 4, one can see that inconsistencies between different reliability measures are found in this study. Apart from $\lambda^{\text {skew }}$, all the other indicators of route 3 are reduced after implementation of road toll. Therefore, we may conclude that the road toll improves the actual travel time reliability of route 3. For route 1 and route 2 , however, the results are opposite.

\subsubsection{Effect on Total Travel Time Distribution and Its Statistics.} In this example, we further investigate the effect of road toll on the total travel time (TTT) distribution and its statistics. From Figure 4, we can observe the following.

(i) The TTT distributions corresponding to the toll and the toll-free cases follow a lognormal distribution. However, the TTT distribution corresponding to the toll case is reduced (shifted to the left) compared to that of the toll-free case. This result indicates that road tolls can encourage road users to make their travel behavior choices based on the full costs they inflict upon society, and thus reduce the TTT of road network.

(ii) In this example, compared to the toll case, the probability distribution of the TTT under the toll-free case is more skewed and wide spread (larger values of $\lambda^{\text {skew }}$ and $\lambda^{\mathrm{var}}$ mean lower reliability of TTT). This may be because the links with lower travel time reliability will induce higher road tolls; the excess traffic flow on the lower reliability links is then transferred to the higher reliability links, so that the reliability of the TTT may be increased. This result implies that the road toll plays an important role in the reliability of the TTT variability.

4.3. Effect of Travel Demand Level and the VoR Level on the Components of Road Toll. In this example, we use a mediumscale traffic network to further demonstrate the effect of travel demand level $z$ and VoR level $\rho$ on the components of road toll. Figure 5 shows a network consisting of fourteen nodes and twenty-one directed links. There are two O-D pairs; one is from node 1 to 12 and the other one is from node 1 to 14 . The free-flow travel times of links 4,10 , and 5 are $4.5,4.5$, and 7.5 minutes, respectively. The free-flow travel times of all the other links are 3 minutes. The capacities of links 5, 7, 6, 12, and 15 are $10,13.3,16.7,16.7$, and 23.3 (veh/min), respectively. The capacities of links 4 and $8-10$ are all 30 (veh/min). The capacities of links $1-3,17,11,14,16,18$, and 19 are all 33.3 (veh/min). The capacities of links 13, 20, and 21 are 43.3 , 66.7 , and $66.7(\mathrm{veh} / \mathrm{min})$, respectively. In order to test the effects of different demand levels, the potential mean total demands for O-D pairs 1 and 2 are set as $\bar{q}^{1}=3800 z$ and $\bar{q}^{2}=4200 z$, respectively. $0 \leq z \leq 1, z$ is the O-D demand multiplier. The VMR of O-D demand is assumed to be 1.5.

Figure 6 displays the effect of demand and the VoR level variations on the congestion pricing on link 4 , when the equilibrium flow pattern is attained. Recall that the PRSNMCP can be decomposed into two individual components, that is, the mean travel time-related toll (MTTRT) and the buffer travel time-related toll (BTTRT). As shown in Figure 6, when the VoR level $\rho=0$, travelers are not sensitive to the travel time variations and the PRSN-MCP model is reduced to a risk-neutral case. Therefore, the BTTRT is equal to zero and the value of the road toll is equal to the MTTRT. In addition, Figure 6 shows that the road toll (BTTRT + MTTRT) and the BTTRT increase with the demand level and the VoR level. However, the MTTRT increases with the demand level and decreases with the travel time reliability 

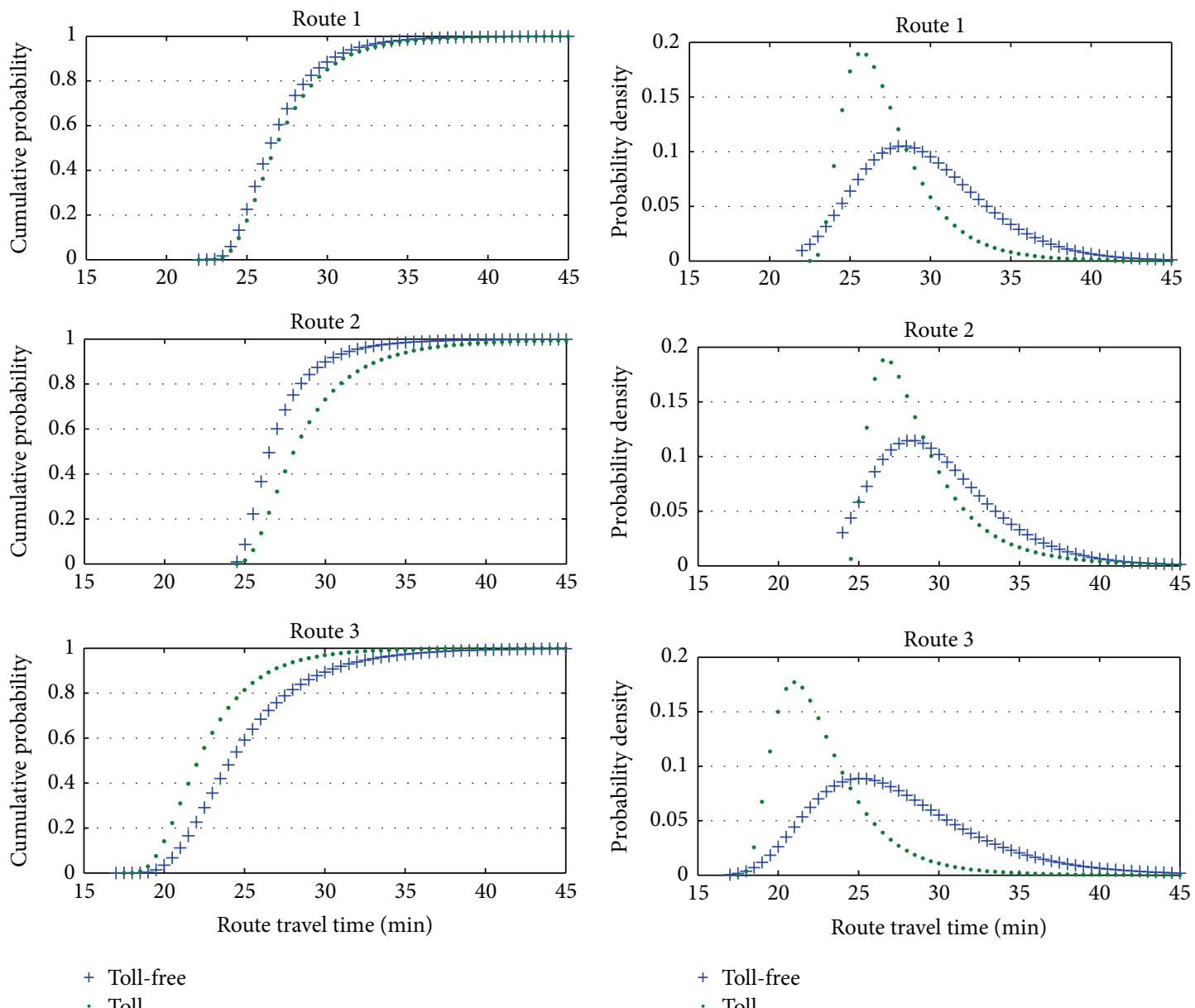

(a)

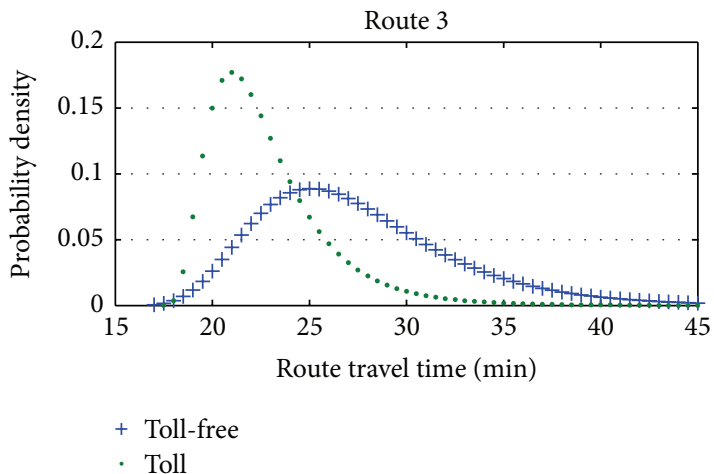

(b)

FIGURE 3: Effect of road toll on the actual travel time distribution.

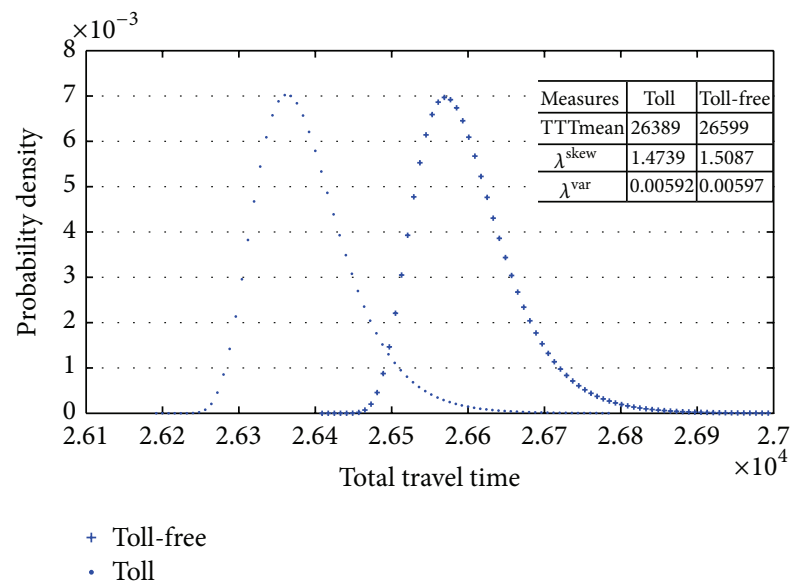

FIGURE 4: Effect of road toll on the total travel time distribution.

confidence level. Perhaps this is because when OD demand is fixed and the VoR level becomes larger, the TTB of link 4 becomes larger as well. Due to the increase of TTB, fewer travelers will choose this link, and the mean link travel time

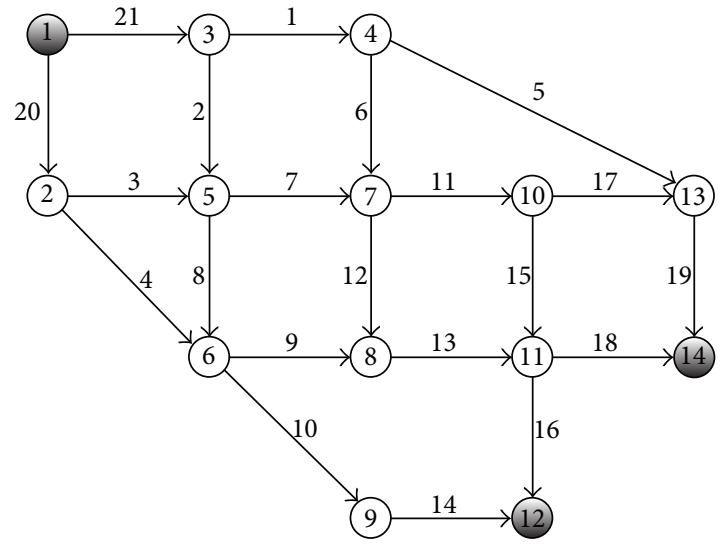

Figure 5: Traffic network.

will get smaller. Thus, the MTTRT will decrease. However, in order to ensure that travelers who choose this link for travel will arrive at their destinations on time and with a higher reliability, it must levy a larger toll on this link. This means that along with the increase of the travelers' VoR level, 


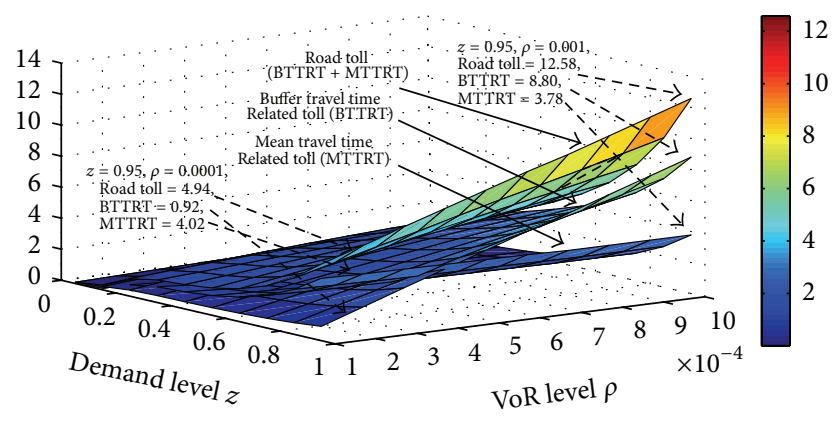

FIGURE 6: Road toll under different OD demand multipliers and value of reliability (VoR) levels.

the difference between the PRSN-MCP and the traditional marginal cost pricing will also increase. To summarize, the higher the VoR level that travelers are concerned with, the worse the actual effect of the traditional marginal cost pricing model.

\section{Conclusions and Future Research}

To make road pricing more efficient and effective, this paper developed a perceived risk-based stochastic networkmarginal cost pricing (PRSN-MCP) model. The new model explicitly accounts for both stochastic demand of road network and stochastic perception errors (SPEs) within the travelers' route choice decision process. The SPE is conditionally dependent on the actual travel time distribution, which is different from the deterministic perception error commonly adopted in the traditional logit-based stochastic user equilibrium (SUE) models by simply adding a random error term such as the Gumbel distribution to the route costs. Using the conditional moment analysis method, we derived a link-based analytical function of PRSN-MCP.

Based on an integrated method combining the moment analysis approach, the fitting distribution method, and the reliability measures, numerical examples with two small networks were presented to illustrate (1) the effect of SPE on the perceived travel time distribution and the components of road toll; (2) the effect of road toll on the actual travel time distribution and its reliability measures; (3) the effect of road toll on the total network travel time distribution and its statistics; and (4) the effect of travel demand level and the VoR level on the components of road toll. The analysis results indicate the importance of explicitly considering both the travel time reliability and the SPE in the road toll model under stochastic traffic network. Ignoring the effect of travel time reliability and SPE may significantly reduce the performance of the first-best MCP tolls, especially under high travelers' confidence and network congestion levels.

Various directions for future research are worth noting. In order to facilitate the presentation of the essential ideas, the link travel times are assumed to be independent of each other in this paper. Therefore, a method for relaxing this assumption would be of interest for further study. Moreover, the capacity random degradation characteristic is not considered in this paper; how to include both stochastic travel demand and stochastic link capacity is another possible extension. Finally, in order to apply the proposed model to a large-scale network, a more efficient solution algorithm needs to be developed, based on column generation procedure.

\section{Appendices}

\section{A. Derivation of the Mean and the Variance of the Perceived Travel Time}

Appendix A provides the derivation of the mean and the variance of the perceived travel time. According to assumption (A4), the perception error for unit travel time, denoted by $\left.\varepsilon\right|_{t=1}$, is a sample from $N\left(\chi, \omega^{2}\right)$. Furthermore, travel time on link $a$ is the sum of independent unit travel times (see assumption (A5)). Therefore, the conditional perception error for link $a$ with deterministic travel time $t_{a}^{0}$ is normally distributed as

$$
\left.\varepsilon_{a}\right|_{T_{a}=t_{a}^{0}} \sim N\left(\chi t_{a}^{0}, \omega^{2} t_{a}^{0}\right)
$$

with conditional moment-generating function (MGF)

$$
\begin{aligned}
\left.M_{\varepsilon_{a}}\right|_{T_{a}=t_{a}^{0}}(s) & =\exp \left(\chi t_{a}^{0} s+\frac{\omega^{2} t_{a}^{0} s^{2}}{2}\right) \\
& =\exp \left[s t_{a}^{0}\left(\chi+\frac{\omega^{2} s}{2}\right)\right],
\end{aligned}
$$

where $s$ is a real number. Following Mirchandani and Soroush [3], the MGF of the perceived travel time $\widetilde{T}_{a}$ of link $a$ for an individual traveler can be derived as follows:

$$
\begin{aligned}
M_{\widetilde{T}_{a}}(s) & =E\left[\exp \left(s \widetilde{T}_{a}\right)\right]=E\left[\exp s\left(T_{a}+\varepsilon_{a}\right)\right] \\
& =E\left\{\exp \left(s T_{a}\right) E_{\left.\varepsilon_{a}\right|_{T_{a}}}\left[\exp \left(\left.s \varepsilon_{a}\right|_{T_{a}}\right)\right]\right\} \\
& =E_{T_{a}}\left\{\exp \left(s T_{a}\right) M_{\left.\varepsilon_{a}\right|_{T_{a}}}(s)\right\},
\end{aligned}
$$

where $E_{x}[]$ denotes the expectation with respect to random variable $x$. Substituting (A.2) in (A.3), we can get

$$
\begin{aligned}
M_{\widetilde{T}_{a}}(s) & =E_{T_{a}}\left\{\exp \left[s T_{a}\left(1+\chi+\frac{\omega^{2} s}{2}\right)\right]\right\} \\
& =M_{T_{a}}\left[s\left(1+\chi+\frac{\omega^{2} s}{2}\right)\right] .
\end{aligned}
$$

From the first derivative of the equation above and evaluating at $s=0$, we can obtain the first moment of the perceived travel time distribution as follows:

$$
E\left[\widetilde{T}_{a}\right]=(1+\chi) E\left[T_{a}\right],
$$

where $E\left[T_{a}\right]$ denotes the mean of the random travel time. Likewise, the second-order moment is derived from the second derivative evaluated at $s=0$,

$$
E\left[\left(\widetilde{T}_{a}\right)^{2}\right]=(1+\chi)^{2} E\left[\left(T_{a}\right)^{2}\right]+\omega^{2} E\left[T_{a}\right]
$$


The variance of the perceived travel time can be expressed as follows:

$$
\begin{aligned}
\operatorname{Var}\left[\widetilde{T}_{a}\right]= & E\left[\left(\widetilde{T}_{a}\right)^{2}\right]-E\left[\widetilde{T}_{a}\right]^{2}=(1+\chi)^{2} \operatorname{Var}\left[T_{a}\right] \\
& +\omega^{2} E\left[T_{a}\right] .
\end{aligned}
$$

\section{B. Derivation of the Mean and the Variance of $\widetilde{T} \widetilde{T}$}

Appendix B provides the derivation of the mean and the variance of $\widetilde{T} \widetilde{T}$. The MGF of $\widetilde{T} \widetilde{T}$ can be represented as follows:

$$
\begin{aligned}
M_{\widetilde{T} \widetilde{T}}(s) & =\sum_{a \in A} E\left[\exp \left(s V_{a} \widetilde{T}_{a}\right)\right] \\
= & \sum_{a \in A} E\left\{\exp \left[s V_{a}\left(T_{a}+\varepsilon_{a}\right)\right]\right\} \\
= & \sum_{a \in A} E_{T_{a}}\left\{\exp \left(s V_{a} T_{a}\right) \exp \left(s V_{a} \varepsilon_{a}\right)\right\} \\
= & \sum_{a \in A} E_{T_{a}}\left\{\exp \left(s V_{a} T_{a}\right) E_{\left.\varepsilon_{a}\right|_{T_{a}}}\left[\exp \left(\left.s V_{a} \varepsilon_{a}\right|_{T_{a}}\right)\right]\right\} \\
= & \sum_{a \in A} E_{T_{a}}\left\{\exp \left(s V_{a} T_{a}\right) M_{\left.\varepsilon_{a}\right|_{T_{a}}}\left(s V_{a}\right)\right\} \\
= & \sum_{a \in A} E_{T_{a}}\left\{\exp \left(s V_{a} T_{a}\right) \exp \left[s V_{a} T_{a}\left(\chi+\frac{\omega^{2} s V_{a}}{2}\right)\right]\right\} .
\end{aligned}
$$

The first-order moment is, from the first derivative evaluated at $s=0$,

$$
E[\widetilde{T} \widetilde{T}]=\sum_{a \in A}(1+\chi) E\left[V_{a} T_{a}\right]
$$

Similarly, the second-order moment of $\widetilde{T} \widetilde{T}$ can be derived from the second derivative evaluated at $s=0$,

$$
E\left[(\widetilde{T} \widetilde{T})^{2}\right]=\sum_{a \in A}\left\{(1+\chi)^{2} E\left[\left(V_{a} T_{a}\right)^{2}\right]+\omega^{2} E\left[V_{a}^{2} T_{a}\right]\right\} .
$$

Then, we can obtain the variance of $\widetilde{T} \widetilde{T}$ as follows:

$$
\begin{aligned}
& \operatorname{Var}[\widetilde{T} \widetilde{T}]= E\left[(\widetilde{T} \widetilde{T})^{2}\right]-E[\widetilde{T} \widetilde{T}]^{2} \\
&=\sum_{a \in A}\left\{(1+\chi)^{2}\left\{E\left[\left(V_{a} T_{a}\right)^{2}\right]-E\left[V_{a} T_{a}\right]^{2}\right\}\right. \\
& \\
&\left.\quad+\omega^{2} E\left[V_{a}^{2} T_{a}\right]\right\} \\
&=\sum_{a \in A}\left\{(1+\chi)^{2} \operatorname{Var}[\mathrm{TT}]+\omega^{2} E\left[V_{a}^{2} T_{a}\right]\right\} .
\end{aligned}
$$

C. Derivation of $E\left[T_{a}\right], \operatorname{Var}\left[T_{a}\right], \partial E[\mathrm{TT}] / \partial v_{\mathrm{a}}$, $\partial \operatorname{Var}[\mathrm{TT}] / \partial v_{\mathrm{a}}$, and $\partial E\left[V_{a}^{2} T_{a}\right] / \partial v_{a}$

Appendix C provides the detailed derivation of $E\left[T_{a}\right]$, $\operatorname{Var}\left[T_{a}\right], \partial E[\mathrm{TT}] / \partial v_{a}, \partial \operatorname{Var}[\mathrm{TT}] / \partial v_{a}$, and $\partial E\left[V_{a}^{2} T_{a}\right] / \partial v_{a}$ term by term.

Using (20) and the BPR function of link travel time, we can easily derive the mean and variance of the link travel time as follows:

$$
E\left[T_{a}\right]=t_{a}^{0}+\frac{\beta t_{a}^{0}}{C_{a}^{n}}\left(v_{a}^{n} y_{a}^{n^{2}-n}\right)
$$

$$
\operatorname{Var}\left[T_{a}\right]=\frac{\beta^{2}\left(t_{a}^{0}\right)^{2}}{C_{a}^{2 n}}\left[v_{a}^{2 n} y_{a}^{4 n^{2}-2 n}-\left(v_{a}^{n} y_{a}^{n^{2}-n}\right)^{2}\right] .
$$

The expected total travel time is given by

$$
\begin{aligned}
E[\mathrm{TT}] & =E\left[\sum_{a \in A} V_{a} T_{a}\right] \\
& =\sum_{a \in A}\left\{t_{a}^{0} E\left[V_{a}\right]+\frac{\beta t_{a}^{0}}{C_{a}^{n}} E\left[V_{a}^{n+1}\right]\right\} \\
& =\sum_{a \in A}\left\{t_{a}^{0} v_{a}+\frac{\beta t_{a}^{0}}{C_{a}^{n}}\left(v_{a}^{n+1} y_{a}^{n^{2}+n}\right)\right\} .
\end{aligned}
$$
yields

$$
\begin{aligned}
& \frac{\partial E[\mathrm{TT}]}{\partial v_{a}} \\
& \quad=t_{a}^{0}+\frac{\beta t_{a}^{0}}{C_{a}^{n}}\left[\frac{n v_{a}^{n-1}\left(1-y_{a}^{2}\right)}{2 y_{a}^{2}}+1\right]\left[(n+1) v_{a} y_{a}^{n^{2}+n}\right] .
\end{aligned}
$$

With (C.3), the variance of the total travel time is expressed as

$$
\begin{aligned}
& \operatorname{Var}[\mathrm{TT}]=\operatorname{Var}\left[\sum_{a \in A}\left(V_{a} \cdot t_{a}^{0}\left(1+\beta\left(\frac{V_{a}}{C_{a}}\right)^{n}\right)\right)\right] \\
& =\operatorname{Var}\left[\sum_{a \in A}\left(V_{a} \cdot t_{a}^{0}+\frac{\beta t_{a}^{0}}{C_{a}^{n}} V_{a}^{n+1}\right)\right] \\
& =\sum_{a \in A}\left\{\operatorname{Var}\left[V_{a} \cdot t_{a}^{0}\right]+\operatorname{Var}\left[\frac{\beta t_{a}^{0}}{C_{a}^{n}} V_{a}^{n+1}\right]\right. \\
& \left.+2 \operatorname{Cov}\left(V_{a} \cdot t_{a}^{0}, \frac{\beta t_{a}^{0}}{C_{a}^{n}} V_{a}^{n+1}\right)\right\} \\
& =\sum_{a \in A}\left\{\left(t_{a}^{0}\right)^{2} \cdot \operatorname{Var}\left[V_{a}\right]+\left(\frac{\beta t_{a}^{0}}{C_{a}^{n}}\right)^{2} \operatorname{Var}\left[V_{a}^{n+1}\right]\right. \\
& \left.+\frac{2 \beta\left(t_{a}^{0}\right)^{2}}{C_{a}^{n}}\left\{E\left[V_{a}^{n+2}\right]-E\left[V_{a}^{n+1}\right] E\left[V_{a}\right]\right\}\right\}
\end{aligned}
$$




$$
\begin{aligned}
=\sum_{a \in A}\{( & \left.t_{a}^{0}\right)^{2} \cdot \mathrm{VMR} \cdot v_{a}+\left(\frac{\beta t_{a}^{0}}{C_{a}^{n}}\right)^{2} \\
& \times\left\{v_{a}^{2 n} y_{a}^{4 n^{2}+6 n+2}-\left(v_{a}^{n+1} y_{a}^{n^{2}+n}\right)^{2}\right\} \\
& \left.+\frac{2 \beta\left(t_{a}^{0}\right)^{2}}{C_{a}^{n}} v_{a}^{n+2} y_{a}^{n^{2}+n}\left(y_{a}^{2 n+2}-1\right)\right\} .
\end{aligned}
$$

Differentiating (C.5) with respect to the mean link flow $v_{a}$ yields

$$
\begin{aligned}
& \frac{\partial \operatorname{Var}[\mathrm{TT}]}{\partial v_{a}} \\
& =\left(t_{a}^{0}\right)^{2} \cdot \mathrm{VMR}+\left(\frac{\beta t_{a}^{0}}{C_{a}^{n}}\right)^{2} \\
& \times\left\{\left\{v_{a}^{2 n} y_{a}^{4 n^{2}+6 n}\right.\right. \\
& \left.\times\left[(2 n+2) v_{a}-\left(2 n^{2}+n-1\right) \cdot \mathrm{VMR}\right]\right\} \\
& -\left\{v_{a}^{2 n} y_{a}^{2 n^{2}+2 n-2}\right. \\
& \left.\left.\times\left[(2 n+2) v_{a}-\left(n^{2}-n-2\right) \cdot \mathrm{VMR}\right]\right\}\right\} \\
& +\frac{2 \beta\left(t_{a}^{0}\right)^{2}}{C_{a}^{n}} \\
& \times\left\{\left\{v_{a}^{n} y_{a}^{n^{2}+3 n}\right.\right. \\
& \left.\times\left[(n+2) v_{a}-\frac{\left(n^{2}+n-2\right)}{2} \cdot \operatorname{VMR}\right]\right\} \\
& -\left\{v_{a}^{n} y_{a}^{n^{2}+n-2}\right. \\
& \left.\left.\times\left[(n+2) v_{a}-\frac{\left(n^{2}-n-4\right)}{2} \cdot \mathrm{VMR}\right]\right\}\right\} .
\end{aligned}
$$

Using (19), we have

$$
\begin{aligned}
E\left[\left(V_{a}\right)^{2} T_{a}\right] & =t_{a}^{0} E\left[V_{a}\right]^{2}+\frac{\beta t_{a}^{0}}{C_{a}^{n}} E\left[V_{a}^{n+2}\right] \\
& =t_{a}^{0} v_{a}^{2} y_{a}^{2}+\frac{\beta t_{a}^{0}}{C_{a}^{n}}\left(v_{a}^{n+2} y_{a}^{n^{2}+3 n+2}\right)
\end{aligned}
$$

Differentiating (C.7) with respect to the mean link flow $v_{a}$ and performing some simple algebraic operations, we have

$$
\begin{aligned}
\frac{\partial E\left[\left(V_{a}\right)^{2} T_{a}\right]}{\partial v_{a}}= & 2 \cdot t_{a}^{0} v_{a} y_{a}^{2}-t_{a}^{0} \cdot \mathrm{VMR} \\
+\frac{\beta t_{a}^{0}}{C_{a}^{n}}[ & (n+2) v_{a}^{n+1} y_{a}^{n^{2}+3 n+2} \\
& \left.\quad-\frac{n^{2}+3 n+2}{2} \cdot \mathrm{VMR} \cdot v_{a}^{n} y_{a}^{n^{2}+3 n}\right] .
\end{aligned}
$$

\section{Acknowledgments}

This research has been supported by Humanities and Social Sciences Youth Foundation of the Ministry of Education of China (Project no. 12YJCZH309) and Specialized Research Fund for the Doctoral Program of Higher Education (Project no. 20120041120006). The authors appreciate the efforts of Dr. Xiantao Xiao during the initial phase of this investigation.

\section{References}

[1] H. Yang, "System optimum, stochastic user equilibrium, and optimal link tolls," Transportation Science, vol. 33, no. 4, pp. 354360, 1999.

[2] M. Maher, K. Stewart, and A. Rosa, "Stochastic social optimum traffic assignment," Transportation Research B, vol. 39, no. 8, pp. 753-767, 2005.

[3] P. Mirchandani and H. Soroush, "Generalized traffic equilibrium with probabilistic travel times and perceptions," Transportation Science, vol. 21, no. 3, pp. 133-152, 1987.

[4] T. Yao, T. L. Friesz, M. M. Wei, and Y. Yin, "Congestion derivatives for a traffic bottleneck," Transportation Research B, vol. 44, no. 10, pp. 1149-1165, 2010.

[5] M. A. Abdel-Aty, R. Kitamura, and P. P. Jovanis, "Using stated preference data for studying the effect of advanced traffic information on drivers' route choice," Transportation Research C, vol. 5, no. 1, pp. 39-50, 1997.

[6] D. Brownstone and K. A. Small, "Valuing time and reliability: assessing the evidence from road pricing demonstrations," Transportation Research A, vol. 39, no. 4, pp. 279-293, 2005.

[7] H. X. Liu, W. Recker, and A. Chen, "Uncovering the contribution of travel time reliability to dynamic route choice using realtime loop data," Transportation Research A, vol. 38, no. 6, pp. 435-453, 2004.

[8] Y. Asakura and M. Kashiwadani, "Road network reliability caused by daily fluctuation of traffic flow," in Proceedings of the 19th PTRC Summer Annual Meeting, pp. 73-84, Brighton, UK, 1991.

[9] A. Nicholson, J. D. Schmocker, and M. G. H. Bell, "Assessing transport reliability: malvolence and user knowledge," in The Network Reliability of Transport: Proceedings of the 1st International Symposium on Transportation Network Reliability, M. G. H. Bell and Y. Iida, Eds., Pergamon, London, UK, 2003.

[10] A. Chen and Z. Zhou, "Chapter 7: a stochastic $\alpha$-reliable meanexcess traffic Equilibrium model with probabilistic travel times and perception errors," in Proceedings of the 18th International 
Symposium of Transportation and Traffic Theory, pp. 117-145, Springer, 2009.

[11] T. Uchida and Y. Iida, "Risk assignment: a new traffic assignment model considering the risk of travel time variation," in Proceedings of the 12th International Symposium on Transportation and Traffic Theory, C. F. Daganzo, Ed., pp. 89-105, Amsterdam, The Netherlands, 1993.

[12] S. D. Boyles, K. M. Kockelman, and S. Travis Waller, "Congestion pricing under operational, supply-side uncertainty," Transportation Research C, vol. 18, no. 4, pp. 519-535, 2010.

[13] A. Sumalee and W. Xu, "First-best marginal cost toll for a traffic network with stochastic demand," Transportation Research $B$, vol. 45, no. 1, pp. 41-59, 2011.

[14] L. M. Gardner, S. D. Boyles, and S. T. Waller, "Quantifying the benefit of responsive pricing and travel information in the stochastic congestion pricing problem," Transportation Research A, vol. 45, no. 3, pp. 202-218, 2011.

[15] A. Chen, Z. Ji, and W. Recker, "Travel time reliability with risksensitive travelers," Transportation Research Record, no. 1783, pp. 27-33, 2002.

[16] Z. Zhou and A. Chen, "Comparative analysis of three user equilibrium models under stochastic demand," Journal of Advanced Transportation, vol. 42, no. 3, pp. 239-263, 2008.

[17] A. Nagurney, Network Economics: A Variational Inequality Approach, Kluwer Academic Publishers, Boston, Mass, USA, 1999.

[18] H. Shao, W. H. K. Lam, and M. L. Tam, "A reliability-based stochastic traffic assignment model for network with multiple user classes under uncertainty in demand," Networks and Spatial Economics, vol. 6, no. 3-4, pp. 173-204, 2006.

[19] S. Clark and D. Watling, "Modelling network travel time reliability under stochastic demand," Transportation Research B, vol. 39, no. 2, pp. 119-140, 2005.

[20] I. D. Hill, R. Hill, and R. L. Holder, "Algorithm AS 99: fitting Johnson curves by moments," Applied Statistics, vol. 25, no. 2, pp. 180-189, 1976.

[21] X. Xu, A. Chen, and L. Cheng, "Assessing the effects of stochastic perception error under travel time variability," Transportation, vol. 40, no. 3, pp. 525-548, 2012.

[22] A. Chen, J. Kim, Z. Zhou, and P. Chootinan, "Alpha reliable network design problem," Transportation Research Record, vol. 2029, pp. 49-57, 2007.

[23] J. W. C. van Lint and H. J. van Zuylen, "Monitoring and predicting freeway travel time reliability: using width and skew of day-to-day travel time distribution," Transportation Research Record, no. 1917, pp. 54-62, 2005. 


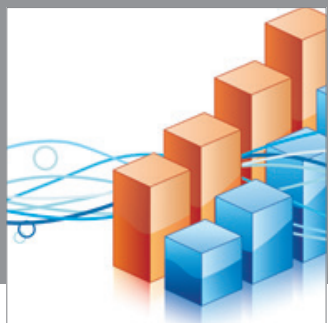

Advances in

Operations Research

mansans

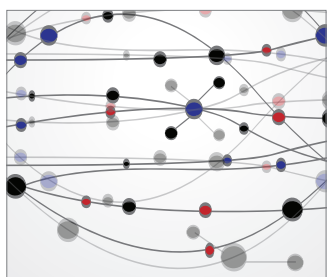

The Scientific World Journal
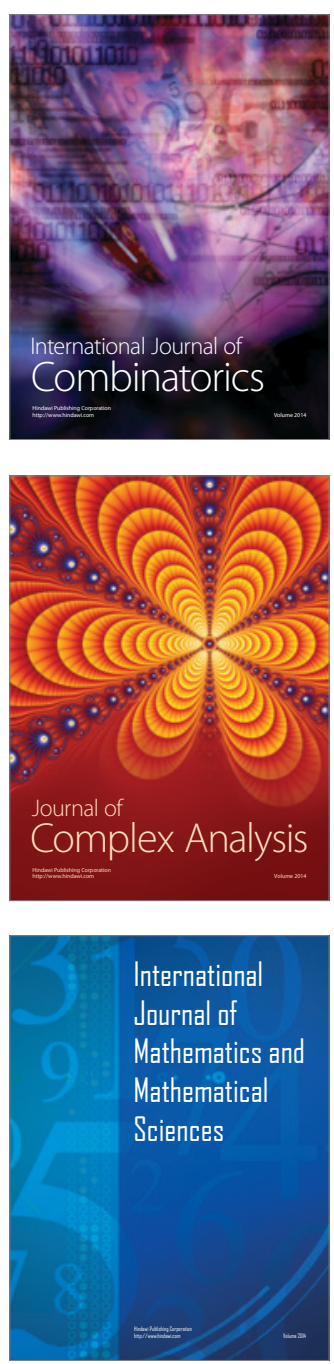
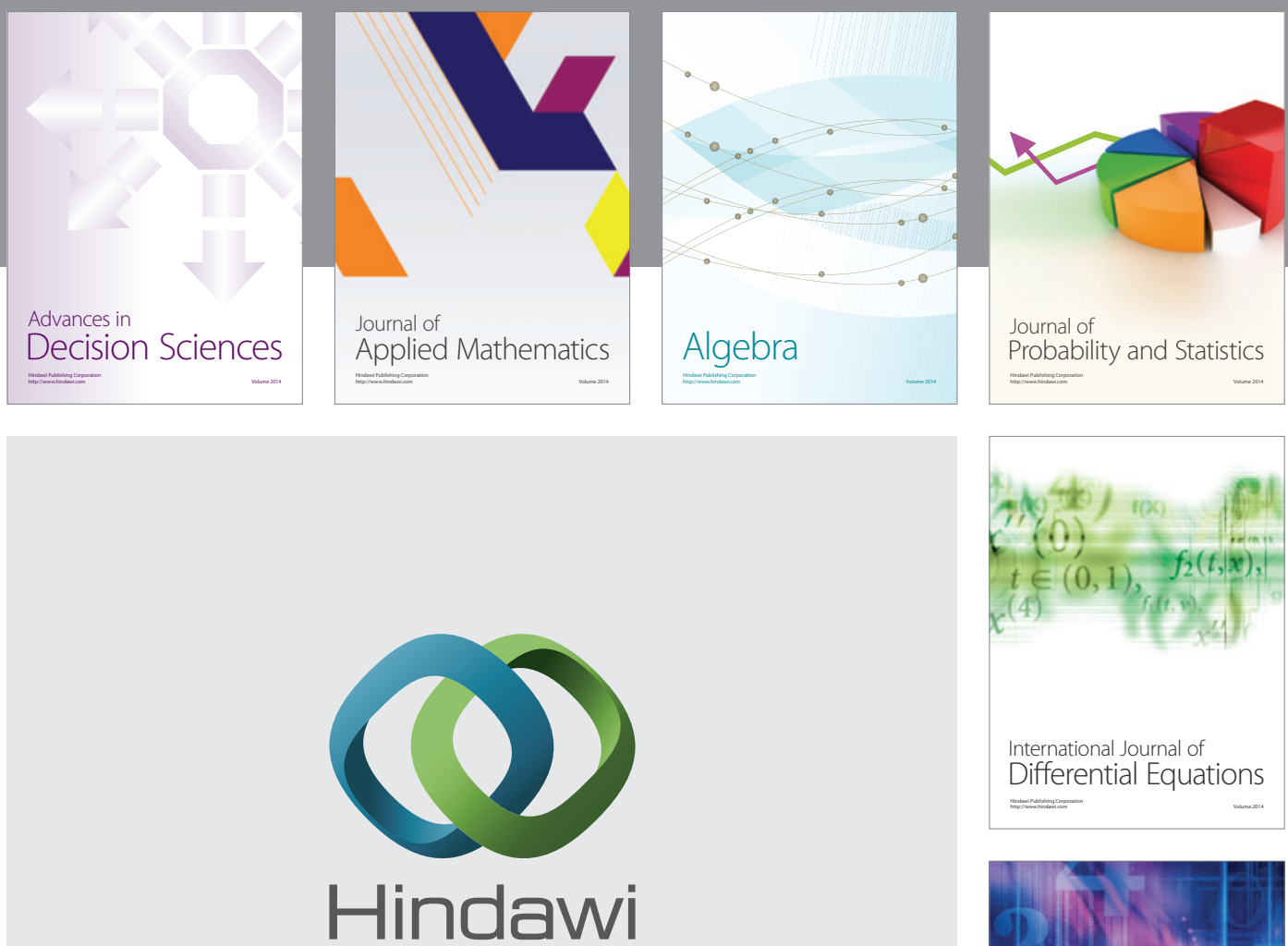

Submit your manuscripts at http://www.hindawi.com
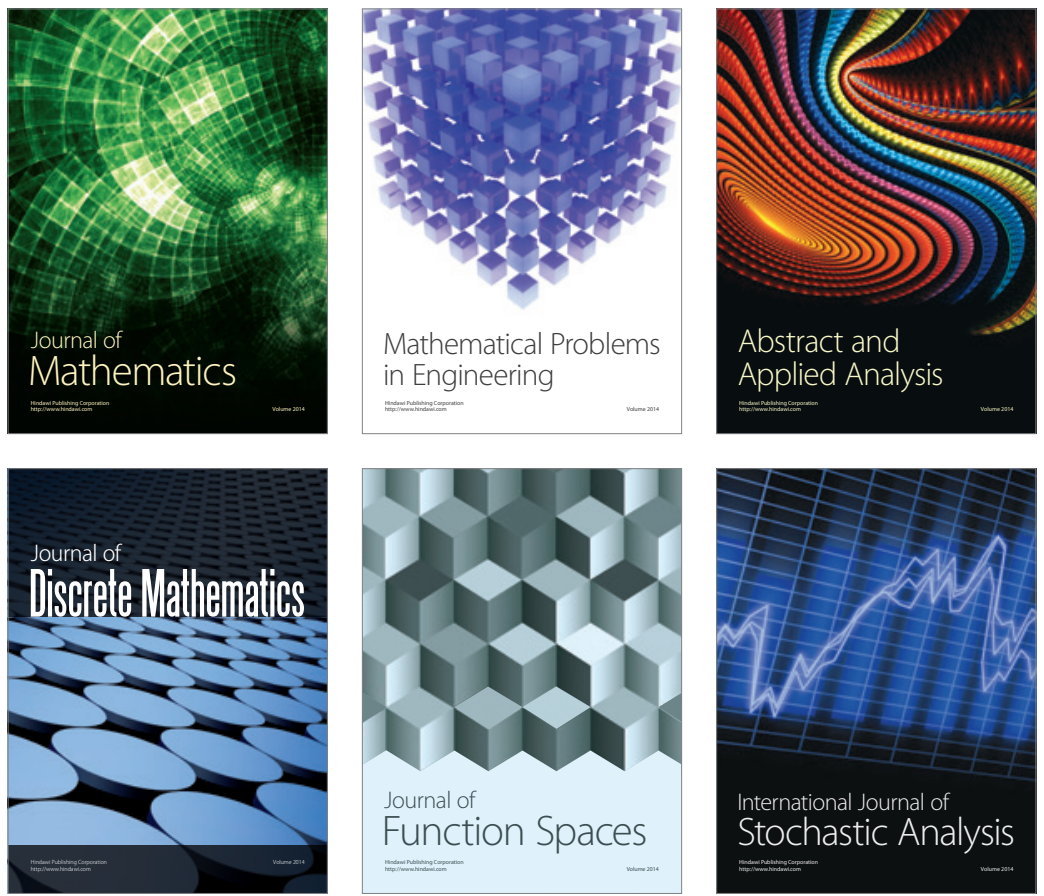

Journal of

Function Spaces

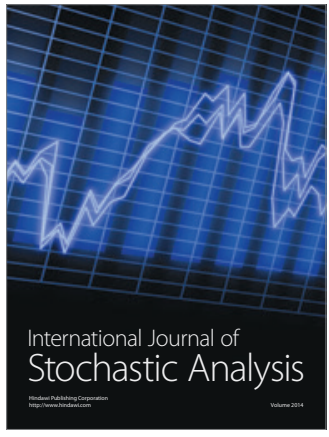

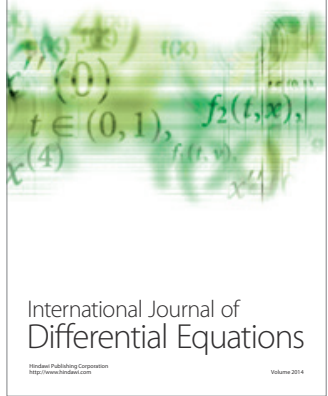
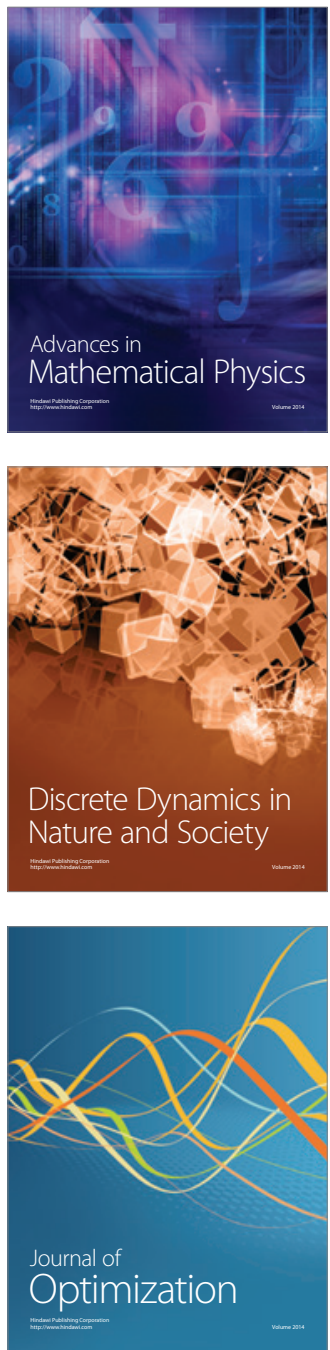